\title{
Efecto neuroprotector del Lepidium meyenii, ecotipo negro (maca negra) en ratas crías frente al daño cerebral inducido por la ingesta de sulfato ferroso y vitamina $\mathrm{C}$ durante la gestación de las ratas madre
}

\author{
Luzmila Troncoso, Emilio Guija, Felio Palomino, Mercedes Soberón, Gisela Oliveira, \\ Marco Núñez, Juana Flores, Sheresnarda Acuña, Carmen Terrazas \\ Centro de Investigación de Bioquímica y Nutrición, Facultad de Medicina, UNMSM. Lima, Perú. \\ Itroncos055@gmail.com
}

Objetivo: Evaluar el efecto neuroprotector del Lepidium meyenii, ecotipo negro en ratas crías por ingesta de sulfato ferroso y vitamina C (SFC) durante la gestación de ratas madre.

Diseño: Experimental, analítico.

Institución: Centro de Investigación de Bioquímica y Nutrición.

Material biológico: Lepidium meyenii, ecotipo negro (maca negra: MN), ratas.

Intervenciones: Se distribuyeron 30 ratas albinas de dos meses de edad, en tres grupos. Tratamiento en los últimos 7 días de gestación: grupo A, SFC (1 mg de hierro elemental y $10 \mathrm{mg}$ de vitamina C); grupo B: SFC (1 mg de hierro elemental y $10 \mathrm{mg}$ de vitamina C) y extracto acuoso de MN (1,0 g/kg pc); grupo C: agua destilada (1 mL). Al nacimiento, la mitad de la camada fue sacrificada y el cerebro separado; la otra mitad fue sometida a la prueba de laberinto de agua de Morris, a los 21 días de edad, sacrificada y el cerebro separado.

Principales medidas de resultados: Signos histopatológicos de necrosis cerebral y tiempo (segundos) en la Prueba de Morris.

Resultados: Las ratas del grupo A presentaron hemorragia intercisural severa, homogenización del parénquima, ausencia de núcleo y pérdida de la membrana celular neuroglial, ausencia de células piramidales. Los grupos B y C no mostraron alteraciones. Hubo correspondencia con la Prueba de Morris.

Conclusión: El Lepidium meyenii ecotipo negro (MN) fue neuroprotector cerebral en ratas crías.

Palabras clave: Lepidium meyenii maca negra, neuroprotector, daño cerebral, sulfato ferroso y vitamina C, ratas cría.

Isocromosomas en síndrome de Turner

\author{
Nery Romero, Rafael Vega, Maritza San Martin, Rosario Gamarra \\ Hospital "San Bartolomé". Lima, Perú. \\ neryromerorojas@gmail.com
}

Un isocromosoma se origina,durante la meiosis,por división transversal a nivel del centrómero; de manera que el cromosoma pierde uno de los brazos y el otro brazo se duplica, formándose dos copias del mismo brazo y produciéndose un cromosoma metacéntrico con dos brazos de igual longitud, idénticos, no homólogos, con cromátides hermanas. Los isocromosomas $X$, especialmente el iso(Xq), están presentes en el sindrome de Turner; los isocromosomas autosómicos en su mayor parte son letales. Presentamos en 56 pacientes con sindrome de Turner 14 casos de isocromosomas.

Objetivo: Presentar isocromosomas en sindrome de Turner.

Diseño: Descriptivo, retrospectivo.

Institución: Hospital "San Bartolomé", Lima, Perú.

Participantes: 14 pacientes.

Intervenciones: Cariotipo en sangre periférica.

Principales medidas de resultados: Cariotipo con bandeo G.

Resultados: Entre 2006 y 2013, encontramos 14 casos de isocromosomas en 56 casos de síndrome de Turner, 6 casos con 46,X,i(X) (q10), 5 casos con mos45,X/46,X,i(X)(q10), 1 caso 46,X,i(Xp)(p10), 1 caso 46,X,i(Yq)(q10) y 1 caso mos45,X/idic(X)(p2). La edad de las pacientes osciló entre 2 dias y 51 años; 12 pacientes consultaron por amenorrea primaria, 1 por linfedema de pies y 1 por talla baja.

Conclusiones: Los isocromosomas estuvieron presentes en $25 \%$ de nuestros casos de sindrome de Turner. Las características clínicas no difirieron de los otros cariotipos de Turner.

Palabras clave: Isocromosoma X, isocromosoma $Y$, mos45,X/idic $(X)(p 2)$, síndrome de Turner. 